\title{
WASP-4b transit observations with GROND ${ }^{\star}, \star \star$
}

\author{
N. Nikolov ${ }^{1}$, Th. Henning ${ }^{1}$, J. Koppenhoefer ${ }^{2,3}$, M. Lendl ${ }^{4}$, G. Maciejewski ${ }^{5}$, and J. Greiner ${ }^{3}$ \\ 1 Max-Planck-Institut für Astronomie, Königstuhl 17, 69117 Heidelberg, Germany \\ e-mail: nikolov@mpia.de \\ 2 Universitäts-Sternwarte München, Scheinerstr. 1, 81679 Munich, Germany \\ 3 Max Planck Institute for Extraterrestrial Physics, Geissenbachstr., 85748 Garching, Germany \\ 4 Observatoire de Genève, Université de Genève, 51 chemin des Maillettes, 1290 Sauverny, Switzerland \\ 5 Toruń Centre for Astronomy, Nicolaus Copernicus University, Gagarina 11, 7100 Toruń, Poland
}

Received 26 October 2011 / Accepted 26 January 2012

ABSTRACT

\begin{abstract}
Context. Ground-based simultaneous multiband transit observations allow an accurate system parameter determination and may lead to the detection and characterization of additional bodies via the transit timing variations (TTVs) method.

Aims. We aim to (i) characterize the heavily bloated WASP-4b hot Jupiter and its star by measuring system parameters and the dependence of the planetary radius as a function of four (Sloan $g^{\prime}, r^{\prime}, i^{\prime}, z^{\prime}$ ) wavelengths and (ii) search for TTVs.

Methods. We recorded 987 images during three complete transits with the GROND instrument, mounted on the MPG/ESO-2.2 m telescope at La Silla Observatory. Assuming a quadratic law for the stellar limb-darkening, we derived the system parameters by simultaneous fitting a composite transit light curve over all bandpasses. To compute uncertainties of the fitted parameters, we employed the bootstrap Monte Carlo method.

Results. The three central transit times are measured with precision down to $6 \mathrm{~s}$. We find a planetary radius $R_{\mathrm{p}}=1.413 \pm 0.020 R_{\mathrm{Jup}}$, an orbital inclination $i=88.57 \pm 0.45^{\circ}$ and calculate a new ephemeris, a period $P=1.33823144 \pm 0.00000032$ days and a reference transit epoch $T_{0}=2454697.798311 \pm 0.000046$ (BJD). Analysis of the new transit mid-times in combination with previous measurements shows no sign of a TTV signal greater than $20 \mathrm{~s}$. We perform simplified numerical simulations to place upper-mass limits of a hypothetical perturber in the WASP-4b system.
\end{abstract}

Key words. planets and satellites: fundamental parameters - methods: data analysis - planetary systems

\section{Introduction}

Observations have shown that more than 100 extrasolar planets pass in front of the disk of their parent stars since the first transit observations reported in 2000 by Charbonneau et al. (see also Henry et al. 2000; Mazeh et al. 2000). These close-in planets orbit their host stars with periods typically shorter than $\sim 10$ days, probably formed at greater orbital distances and later migrated inward governed by processes that are still under debate. The discovery of each transiting extrasolar planet is of great interest for planetary science because these objects provide a unique access to an accurate determination of radii (down to a few percent) and masses via transit photometry and radial velocity measurements of the host star (Henry et al. 2000; Charbonneau et al. 2000). Moreover, they allow one to plot thermal maps of the planetary surface via infrared spectra (Richardson et al. 2007; Grillmair et al. 2007; Knutson et al. 2007), determine the planetary temperature profiles, and permit studies of the stellar spinorbit alignment (Queloz et al. 2000; Triaud et al. 2010; Winn et al. 2011; Johnson et al. 2011). All of these form a set of astrophysically important parameters, which are critical for constraining the formation and evolution of these interesting objects.

\footnotetext{
* Based on observations collected with the Gamma Ray burst Optical and Near-infrared Detector (GROND) at the MPG/ESO-2.2 $\mathrm{m}$ telescope at La Silla Observatory, Chile. Programme 083.A-9010.

$\star \star$ Light curves are only available at the CDS via anonymous ftp to cdsarc.u-strasbg.fr (130.79.128.5) or via

http://cdsarc.u-strasbg.fr/viz-bin/qcat?J/A+A/539/A159
}

Furthermore, the time intervals between successive transits are strictly constant if the system consists of a planet moving on a circular orbit around the parent star. If a third body is present in the system, it would perturb the transiting planet and thus cause the time interval between successive transits to vary. The resulting transit timing variations (TTVs) allow the determination of the orbital period and mass of the perturber down to subEarth masses (Miralda-Escudé 2002; Holman \& Murray 2005; Holman et al. 2010; Lissauer et al. 2011).

The transiting planet WASP-4b was discovered by Wilson et al. (2008) within the Wide Angle Search for Planets in the southern hemisphere (WASP-S, Pollacco et al. 2006). The planet is a $1.12 M_{\text {Jup }}$ hot Jupiter that orbits a G7V star with a period of 1.34 day. WASP-4b was found to have a heavily irradiated atmosphere and inflated radius. Refined planetary orbital and physical parameters, based on transit photometry were presented by Gillon et al. (2009), who used the Very Large Telescope (VLT) to observe one transit, Winn et al. (2009), who measured two transits with the Magellan (Baade) $6.5 \mathrm{~m}$ telescope at Las Campanas Observatory, Southworth et al. (2009), who observed four transits using the $1.54 \mathrm{~m}$ Danish Telescope at ESO La Silla Observatory and Sanchis-Ojeda et al. (2011), who observed four transits using the Magellan (Baade) $6.5 \mathrm{~m}$ telescope. During two of the transits, Sanchis-Ojeda et al. (2011) observed a short-lived, low-amplitude anomaly that the authors interpreted as the occultation of a starspot by the planet. Southworth et al. (2009) also noted similar anomalies in the light curves they observed and the possibility that they were caused by starspot 
occultations. Sanchis-Ojeda et al. (2011) combined their data set with that of Southworth et al. (2009) and found that each of them is consistent with a single spot and a star that is well-aligned with the orbit. Tracking this starspot, it was possible to measure the rotation period of the host star. A new possible starspot has recently been detected in two closely spaced transits of WASP-4 by the MiNDSTEp collaboration with the Danish telescope (Mancini 2011, priv. comm.). Furthermore, Beerer et al. (2011) performed space-based secondary eclipse photometry, using the IRAC instrument on the Warm Spitzer Space Telescope, of the planet WASP-4b in the 3.6 and $4.5 \mu \mathrm{m}$ bands. Their data suggest that WASP-4b's atmosphere lacks a strong thermal inversion on the day-side of the planet, which is an unexpected result for a highly irradiated atmosphere. Cáceres et al. (2011) analyzed high-cadence near-infrared ground-based photometry, detected the planet's thermal emission at $2.2 \mu \mathrm{m}$ and concluded that WASP-4b shows an inhomogeneous redistribution of heat from its day- to night-side. Finally, Triaud et al. (2010) investigated the spin-orbit alignment $(\beta)$ of the WASP-4 system by measuring the parent star radial velocity during a transit of its planet (Rossiter-McLaughlin effect) and found $\beta=4_{-34^{\circ}}^{\circ}$, excluding a projected retrograde orbit.

In this paper we present three new transits of WASP-4b observed in August and October 2009 with the GROND instrument (Greiner et al. 2008), attached to the MPG/ESO $2.2 \mathrm{~m}$ telescope at La Silla Observatory. We recorded each transit in four optical $g^{\prime}, r^{\prime}, i^{\prime}$ and $z^{\prime}$ (Sloan) channels simultaneously. Using these new data sets we measured the planet orbital and physical parameters and constrained its ephemeris by simultaneous fitting a new photometric data set over four pass-bands. Unlike all previous observational studies related to the WASP-4b exoplanet, which fitted data in a single bandpass, we modelled a transit light curve using the four bandpasses simultaneously. As pointed out by Jha et al. (2000), this approach permits one to break a fundamental degeneracy in the shape of the transit light curve. Each transit light curve can be described primarily by its depth and duration. For a single-band observations it is always possible to fit these with a larger planet if the stellar radius is also increased and if the orbital inclination is decreased. The main advantage of the multi-band transit photometry is that it allows the determination of the planet orbital inclination unique, independent of any assumptions about the stellar and planetary radii. Finally, we added the three new mid-transit times and searched for TTVs. This paper is organized as follows. In Sect. 2 we present an overview of the three transit observations of WASP-4b and the data reduction. In Sect. 3 we discuss the light curve analysis and the error estimation. Section 4 summarizes the main results.

\section{Observations and data reduction}

Three transits of WASP-4b were observed with the MPG/ESO 2.2-m telescope at La Silla Observatory (Chile) during three runs on UT August 26 and 30 and October 8, 2009 (see Fig. 1). Bad weather conditions prevented data collection during the remaining two nights allocated for the project. To monitor the flux of WASP-4, we used the Gamma Ray burst Optical and Nearinfrared Detector (GROND). This is a gamma-ray burst followup instrument, which allows simultaneous photometric observations in four optical (Sloan $g^{\prime}, r^{\prime}, i^{\prime}, z^{\prime}$ ) and three near-infrared $(J H K)$ bandpasses (Greiner et al. 2008). In the optical channels the instrument is equipped with backside illuminated E2V CCDs $(2048 \times 2048,13.5 \mu \mathrm{m})$. The field of view for each of the four optical channels is $5.4^{\prime} \times 5.4^{\prime}$ with a pixel scale of 0 ' $^{\prime} 158$ pixel $^{-1}$.
During each observing run we verified that WASP-4 and a nearby comparison star were located within GROND's field of view. The comparison star was about $1.3^{\prime}$ southeast from our target. For each run we obtained repeated integrations of WASP-4 and the comparison star for about $3.5 \mathrm{~h}$, bracketing the predicted central transit times. The exposure times were in the range 8-14 s, depending on the weather conditions (i.e. seeing and transparency). During each run we used the fast-readout mode (roughly $10 \mathrm{~s}$ ), achieving a cadence ranging from 18-24 s. To minimize systematic effects in the photometry associated with pixel-to-pixel gain variations we kept the telescope pointing stable. However, owing to technical problems caused by the guiding camera about $35 \%$ of the total number of images were acquired without guiding, which resulted in about 10-12 pixel drifts per observing run (about $3.5 \mathrm{~h}$ ).

In the near-infrared (NIR) channels we obtained images with an integration time of $10 \mathrm{~s}$. Longer exposures are not allowed owing to technical constrain in the GROND instrument. It was found during the analysis that the $J H K$-images were of insufficient signal-to-noise ratio (SNR) to be able to detect the transit and to allow an accurate system parameter derivation from the resulting light curves. In addition, the pixel scale in the NIR detectors is $0.6 \mathrm{px}^{-1}$, which resulted in poorly sampled star point spread functions (psfs) (typically 4-5 pixels). Because we carried out all observations in the NIR without dithering to increase the cadence and to improve the time sampling and the photometry precision, it is hardly possible to stack groups of NIR images with prior background subtraction. Therefore, we excluded the NIR data from the subsequent analysis.

At the beginning of the observations on UT August 26 and 30, 2009, WASP-4 was setting from an air-mass of 1.02 and 1.05, respectively, which increased monotonically to 1.5 and 1.6 at the end of the runs. During the October 8 observations, the air-mass decreased from 1.06 to 1.02 and then increased to 1.15 at the end of the run.

We employed standard IRAF ${ }^{1}$ procedures to perform biasand dark- current subtraction as well as flat-fielding. A median combined bias was computed using 22 zero-second exposure frames and four dark frames were used to compute the master dark. Because a good part of the data was obtained without guiding, it was critical to perform the data reduction with flat-fields of high quality. A master flat-field was calculated using the following methodology. From a set of 12 dithered twilight flats we selected frames with median pixel counts in the range $10 \mathrm{~K}$ to $35 \mathrm{~K}$, which is wellinside the linear regime of the GROND optical detectors. Finally, the reduced (bias-corrected) flat-frames were median-combined to produce master flats for each of the $g^{\prime}, r^{\prime}, i^{\prime}$ and $z^{\prime}$ bands.

Aperture photometry of WASP-4 and the reference star was performed on each calibrated image. To produce the differential light curve of WASP-4, its magnitude was subtracted from that of the comparison star. Fortunately, the latter was of similar color and brightness, which reduced the effect of differential color extinction. For example, the instrumental magnitude differences (comparison star minus WASP-4) measured on UT October 8, 2009 were $\Delta g^{\prime}=0.587 \mathrm{mag}, \Delta r^{\prime}=0.669 \mathrm{mag}$, $\Delta i^{\prime}=0.681 \mathrm{mag}, \Delta z^{\prime}=0.687 \mathrm{mag}$. As pointed out by Winn et al. (2009), the comparison star is slightly bluer than WASP-4 $\left(\Delta\left(g^{\prime}-i^{\prime}\right)=-0.094 \mathrm{mag}\right)$.

1 IRAF is distributed by the National Optical Astronomy Observatories, which are operated by the Association of Universities for Research in Astronomy, Inc., under cooperative agreement with the National Science Foundation. 


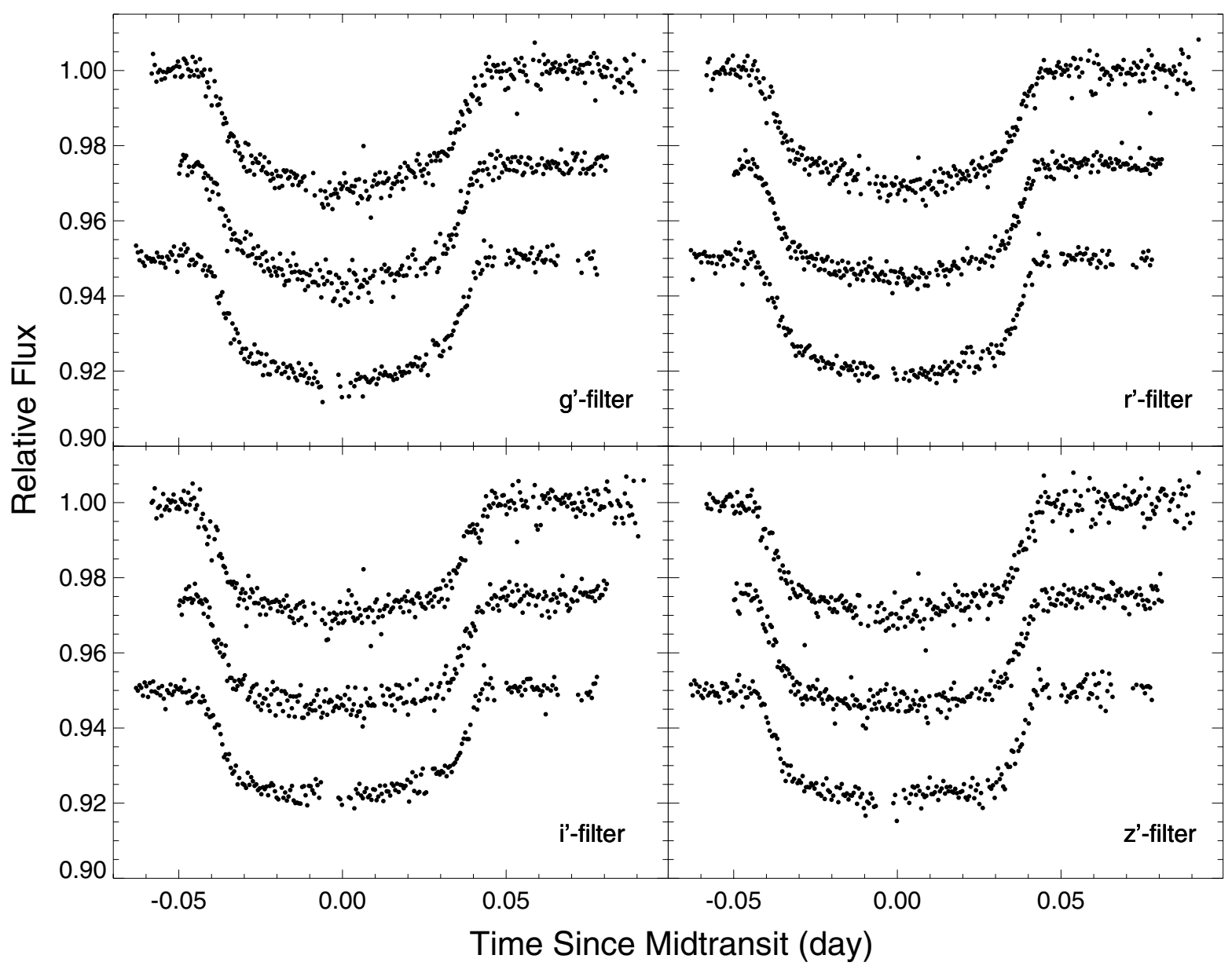

Fig. 1. Relative $g^{\prime}, r^{\prime}, i^{\prime}$ and $z^{\prime}$-band photometry of WASP-4 obtained with GROND. The transits occured (for each panel from top to bottom) on UT August 26, August 30 and October 8, 2009. The transit light curves obtained on each successive run are displayed with an offset of 0.025 in relative flux for a better illustration.

To improve the light curve quality we experimented with various aperture sizes and sky areas, aiming to minimize the scatter of the out-of-transit (OOT) portions, measured by the magnitude root-mean-square (rms). Best results were obtained with aperture radii of 16, 20.5, and 17.5 pixels for UT 2009 August 26, 30 , and October 8 , respectively.

The light curves contain smooth trends, most likely due to differential extinction. To improve its quality we decreased this systematic effect using the OOT portions of our light curves. We plotted the magnitude vs. air-mass data for each run and channel and fit a linear model of the form:

$f(z)=a+b z$

where $(a)$ and $(b)$ are constants and $(z)$ is the air-mass. Once the coefficients were derived, we applied the correction to both the transit and the OOT data. Performing this correction we noticed that the baseline magnitudes of our target and the comparison star show slight, nearly linear correlations with the $(x, y)$ center positions of the PSF centroids on each CCD chip. We removed these near-linear trends from WASP-4 and the reference star by modeling the baseline of the light curve with a polynomial that includes a linear dependency on the centroid center positions $(x$ and $y$ ) of the stars on the detectors using the function of the form

$f(x, y)=1+k_{x} x+k_{y} y+k_{x y} x y$
Table 1. Light curve scatter difference measured with the standard deviation of the time series before and after detrending.

\begin{tabular}{lcccc}
\hline \hline Run & $g^{\prime}$ & $r^{\prime}$ & $i^{\prime}$ & $z^{\prime}$ \\
\hline Aug. 26 P & 0.503 & 0.358 & 0.482 & 0.339 \\
Aug. 30 P & 0.818 & 0.973 & 0.633 & 0.725 \\
Oct. 10 P & 0.733 & 0.614 & 0.430 & 0.495 \\
\hline
\end{tabular}

Notes. All quantities in mmag.

where $\left(k_{x}\right),\left(k_{y}\right)$ and $\left(k_{x y}\right)$ are constants. We then subtracted the fit from the total transit photometry obtained for each channel during the three runs. No other significant trends that were correlated with instrumental parameters were found.

To illustrate the light curve quality improvement we present the scatter decrease after the described detrending procedure (Table 1).

\section{Light curve analysis}

To compute the relative flux of WASP-4 during transit as a function of the projected separation of the planet, we employed the models of Mandel \& Agol (2002), which in addition to the orbital period $P$ and the transit central time $T_{\mathrm{C}}$ are a function of five parameters, including the planet-to-star size ratio $\left(R_{\mathrm{p}} / R_{*}\right.$, where $R_{\mathrm{p}}$ and $R_{*}$ are the absolute values of the planet and star radii), 


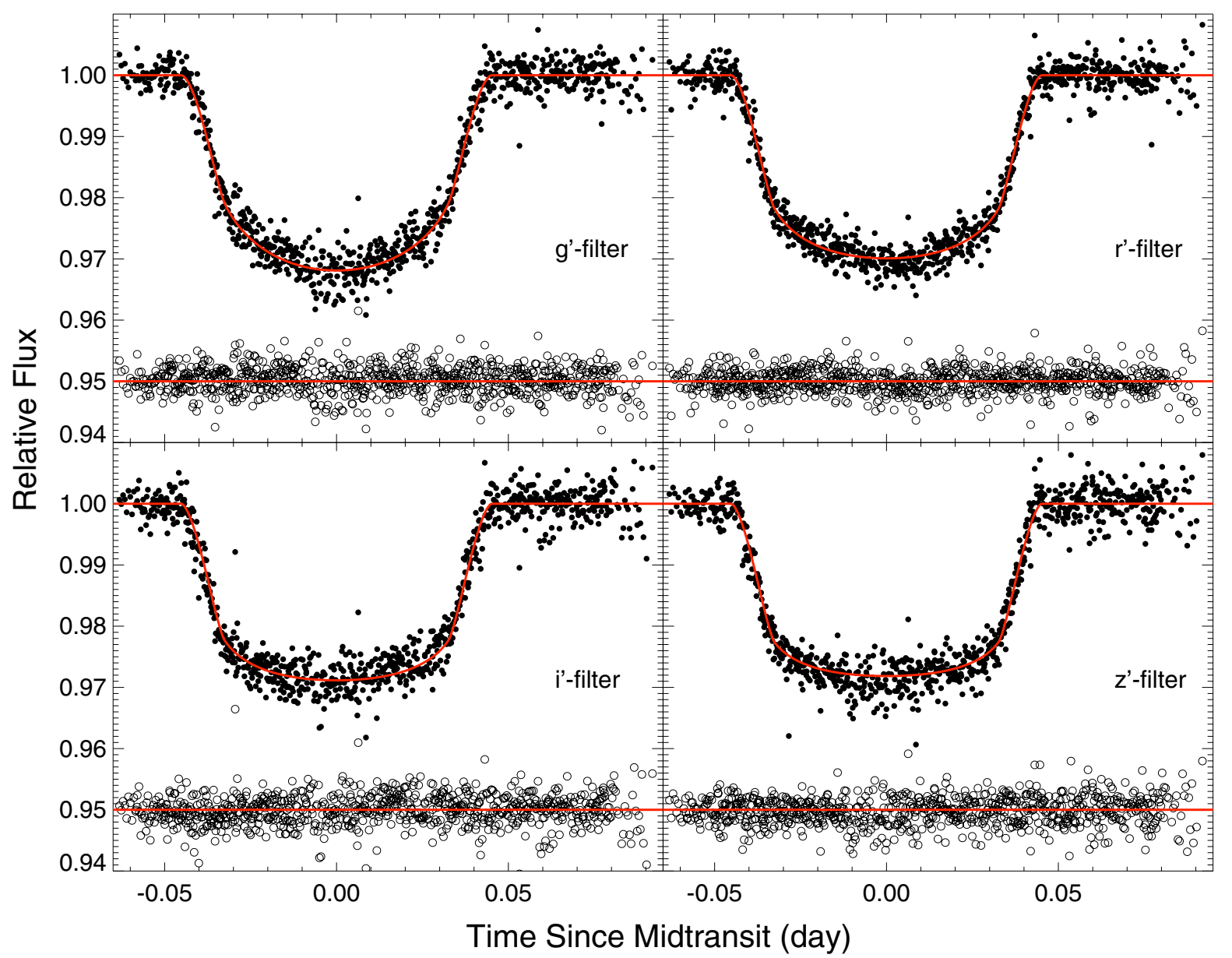

Fig. 2. Composite light curves of WASP-4 obtained during three runs on UT August 26, August 30 and October 8, 2009. The best-fit transit models are superimposed with continuous lines and the observed minus modeled residuals are shown centered at flux level 0.95 on each panel along a constant line.

the orbital inclination $i$, the normalized semimajor axis $\left(a / R_{*}\right.$, where $a$ is the absolute value of the planet semimajor axis) and two limb-darkening coefficients $\left(u_{1}\right.$ and $\left.u_{2}\right)$. Because the parameters $a / R_{*}, R_{\mathrm{p}} / R_{*}$ and $i$ are degenerate in the transit light curve, we assumed fixed values for $M_{*}=0.92 \pm 0.06 M_{\odot}$ from Winn et al. (2009) and $R_{*}=0.907_{+0.014}^{-0.013} R_{*}$ from Sanchis-Ojeda et al. (2011) to break this degeneracy. Ideally we would have liked to fit for all parameters but only the first three determine the best-fit radius of the star, the radius of the planet, its semimajor axis and inclination relative to the observer.

The values for the two limb-darkening coefficients were included in our fitting algorithm to compute the theoretical transit models of Mandel \& Agol (2002). We assume the stellar limbdarkening law to be quadratic,

$\frac{I_{\mu}}{I_{1}}=1-u_{1}(1-\mu)-u_{2}(1-\mu)^{2}$

where $I$ is the intensity and $\mu$ is the cosine of the angle between the line of sight and the normal to the stellar surface. We (initially) fixed the limb-darkening coefficients $\left(u_{1}\right.$ and $\left.u_{2}\right)$ to their theoretical values (see Table 2 ), which we obtained from the calculated and tabulated ATLAS models (Claret 2004). Specifically, we performed a linear interpolation for WASP-4 stellar parameters $T_{\text {eff }}=5500 \pm 100 \mathrm{~K}, \log g=4.4813 \pm 0.0080 \mathrm{cgs}$, $[\mathrm{Fe} / \mathrm{H}]=-0.03 \pm 0.09$ and $v_{\mathrm{t}}=2.0 \pm 1.0 \mathrm{~km} \mathrm{~s}^{-1}$, which we adopted from Winn et al. (2009).
Table 2. Theoretical limb-darkening coefficients (quadratic law).

\begin{tabular}{lcccc}
\hline \hline LD coefficient & $g^{\prime}$ & $r^{\prime}$ & $i^{\prime}$ & $z^{\prime}$ \\
wavelength $(\mathrm{nm})$ & 455 & 627 & 763 & 893 \\
\hline$u_{1}$ (linear) & 0.623 & 0.413 & 0.314 & 0.248 \\
$u_{2}$ (quadratic) & 0.183 & 0.290 & 0.303 & 0.308 \\
\hline
\end{tabular}

To derive the best-fit parameters we constructed a fitting statistic of the form

$\chi^{2}=\sum_{i=1}^{N_{f}}\left[\frac{f_{i}(\text { observed })-f_{i}(\text { predicted })}{\sigma_{i}}\right]^{2}$,

where $f_{i}$ (observed) is the flux of the star observed at the $i$ th moment (with the median of the OOT point normalized to unity), $\sigma_{i}$ controls the weights of the data points and $f_{i}$ (computed) is the predicted value for the flux from the theoretical transit light curve. We assumed the orbital eccentricity to be zero and minimized the $\chi^{2}$ statistic using the downhill simplex routine, as implemented in the IDL AMOEBA function (Press et al. 1992). The minimization method iteratively evaluates the $\chi^{2}$ statistic, yet avoiding derivative estimation until the function converges to the global minimum.

In the entire analysis we derived uncertainties for the fitted parameters using the bootstrap Monte Carlo method 
Table 3. System parameters of the WASP-4 system derived from the light curve analysis.

\begin{tabular}{|c|c|c|c|c|c|}
\hline Parameter & & Value & $68.3 \%$ Conf. limits & Unit & Comment \\
\hline \multicolumn{6}{|l|}{ - Light curve fit- } \\
\hline Planet-to-star size ratio & $p=R_{\mathrm{n}} / R_{*}$ & 0.15655 & 0.00028 & - & 1 \\
\hline Orbital inclination & $i$ & 88.57 & 0.45 & deg & 1 \\
\hline Normalized semimajor axis & $a / R_{*}$ & 5.455 & 0.031 & - & 1 \\
\hline Transit impact parameter & $b=\left(a / R_{*}\right) \cos i$ & 0.136 & 0.043 & - & 1 \\
\hline Reduced chi-square & $\chi_{\text {red }}^{2}$ & 1.005 & - & - & 1 \\
\hline \multicolumn{6}{|l|}{ - Planetary parameters - } \\
\hline Radius & $R_{\mathrm{p}}$ & 1.413 & 0.020 & $R_{\text {Jup }}$ & 2 \\
\hline Semimajor axis & $a$ & 0.02300 & 0.00036 & AU & 2 \\
\hline Mean density & $\rho_{\mathrm{p}}$ & 0.582 & 0.036 & $\rho_{\text {Jup }}$ & 2 \\
\hline Surface gravity & $g_{\mathrm{p}}$ & 15.36 & 0.91 & $\mathrm{~m} \mathrm{~s}^{-2}$ & 2 \\
\hline Transit duration & $t_{\mathrm{D}}$ & 2.1696 & 0.0047 & $\mathrm{~h}$ & 1 \\
\hline Ingress/egress & $t_{\text {ing/egr }}$ & 0.3014 & 0.0039 & $\mathrm{~h}$ & 1 \\
\hline \multicolumn{6}{|l|}{ - Stellar parameters - } \\
\hline Mean density & $\rho_{*}$ & 1.715 & 0.029 & $\mathrm{~g} \mathrm{~cm}^{-3}$ & 1 \\
\hline$g^{\prime}$ Linear limb-darkening coefficient & $u_{1}$ & 0.616 & 0.046 & - & 1 \\
\hline$g^{\prime}$ Quadratic limb-darkening coefficient & $u_{2}$ & 0.211 & 0.060 & - & 1 \\
\hline$r^{\prime}$ Linear limb-darkening coefficient & $u_{1}$ & 0.427 & 0.044 & - & 1 \\
\hline$r^{\prime}$ Quadratic limb-darkening coefficient & $u_{2}$ & 0.303 & 0.064 & - & 1 \\
\hline$i^{\prime}$ Linear limb-darkening coefficient & $u_{1}$ & 0.292 & 0.047 & - & 1 \\
\hline$i^{\prime}$ Quadratic limb-darkening coefficient & $u_{2}$ & 0.304 & 0.060 & - & 1 \\
\hline$z^{\prime}$ Linear limb-darkening coefficient & $u_{1}$ & 0.241 & 0.045 & - & 1 \\
\hline$z^{\prime}$ Quadratic limb-darkening coefficient & $u_{2}$ & 0.386 & 0.063 & - & 1 \\
\hline
\end{tabular}

Notes. (1) Based on the analysis of the multiband light curves presented in this work; (2) values obtained using (1) and results for the $M_{*}, R_{*}, T_{\text {eff }}$ and $M_{\mathrm{p}}$ from Winn et al. (2009) and Sanchis-Ojeda et al. (2011).

(Press et al. 1992). It uses the original data sets (from each run and pass-band), with their $N$ data points to generate synthetic data sets also with the same number of points with replacements. We then fitted each data set to derive parameters. The process was repeated until we obtained an approximately Gaussian distribution for each parameter and took the standard deviation of each distribution as the error of the corresponding fitted parameter.

Ground-based time series data are often compromised with time-correlated noise (e.g. "red noise"; see Pont et al. 2006) and therefore the data weights $\sigma_{i}$ need a special treatment, i.e. the uncertainties must be calculated accurately to obtain reliable estimates of the fitted parameters. It is common practice to use the calculated Poisson noise, or the observed standard deviation of the out-of-transit data for the weights. Our experience shows that these methods often result in underestimated uncertainties of the modeled parameters. To estimate realistic parameter uncertainties we employed two methods. First we rescaled the photometric weights $\sigma_{j}$ so that the best-fitting model for each band and run results in a reduced $\chi^{2}=1$, which therefore requires the initial values for the photometric uncertainties to be multiplied by the factors $\chi_{\text {red }}^{2}=1.396, \chi_{\text {red }}^{2}=1.058$ and $\chi_{\text {red }}^{2}=1.179$, for the UT August 26 and 30 and October 82009 runs, respectively.

Second, we took into account the "red noise" in our data by following the "time-averaging" approach that was proposed by Pont et al. (2006) and used in the transit data analysis of various authors including Gillon et al. (2006), Winn et al. (2007, 2008, 2009) and Gibson et al. (2008). The main idea of the method is to compute the standard deviation (scatter) of the unbinned residuals between the observed and calculated fluxes, $\sigma_{1}$ and also the standard deviation of the time-averaged residuals, $\sigma_{N}$, where the flux of each $N$ data points were averaged creating $M$ bins. In the absence of red noise one would expect

$\sigma_{N}=\frac{\sigma_{1}}{\sqrt{N}} \sqrt{\frac{M}{M-1}}$.

However, in reality $\sigma_{N}$ is larger than $\sigma_{1}$ by some factor $\beta$, which, as pointed out by Winn et al. (2007), is specific to each parameter of the fitted model. For simplicity we assumed that the values of $\beta$ are the same for all parameters and found its value by averaging $\beta$ over a range of bins with timescales consistent with the duration of the transit ingress or egress i.e. $10-30 \mathrm{~min}$. The resulting values for $\beta$ were then used to rescale $\sigma_{j}$ in the $\chi^{2}$ by this value (see Table 4).

To derive transit mid-times $\left(T_{\mathrm{C}}\right)$ we fixed the orbital period $P$ to an initial value taken from Sanchis-Ojeda et al. (2011) and fitted the light curves from each run for the best $T_{\mathrm{C}}$ planet-tostar size ratio, inclination and normalized semimajor axis. We then found the best orbital period $P$ and $T_{\mathrm{C}}$ using the method discussed in Sect. 4.2. Then we took the new values for the $T_{\mathrm{C}}$ and $P$ and repeated the fit for the best planet-to-star size ratio, inclination, and normalized semimajor axis. This procedure was iterated until we obtained a consistent solution.

\section{Results}

\subsection{System parameters}

We set the mass and radius of the star equal to $0.92 \pm$ $0.06 M_{\odot}$ and $0.907_{+0.014}^{-0.013} R_{*}$, respectively, and determined the best-fit radius for WASP- $4 \mathrm{~b}, R_{\mathrm{p}}$ by minimizing the $\chi^{2}$ function over the four band-passes and the three runs simultaneously. 
Table 4. Summary of the residual rms (observed minus calculated flux) of the unbinned and binned data.

\begin{tabular}{lcccc}
\hline \hline Run & Band & $\begin{array}{c}\sigma \\
{[\mathrm{mag}]}\end{array}$ & $\beta$ & $\begin{array}{c}\sigma_{\text {sys }} \\
{[\mathrm{mag}]}\end{array}$ \\
\hline Aug. 26 & $g^{\prime}$ & 0.0025 & 1.41 & 0.0035 \\
Aug. 26 & $r^{\prime}$ & 0.0025 & 1.38 & 0.0035 \\
Aug. 26 & $i^{\prime}$ & 0.0028 & 1.45 & 0.0041 \\
Aug. 26 & $z^{\prime}$ & 0.0028 & 1.49 & 0.0042 \\
Aug. 30 & $g^{\prime}$ & 0.0023 & 1.19 & 0.0027 \\
Aug. 30 & $r^{\prime}$ & 0.0017 & 1.10 & 0.0019 \\
Aug. 30 & $i^{\prime}$ & 0.0025 & 1.23 & 0.0031 \\
Aug. 30 & $z^{\prime}$ & 0.0022 & 1.29 & 0.0028 \\
Oct. 08 & $g^{\prime}$ & 0.0018 & 1.22 & 0.0022 \\
Oct. 08 & $r^{\prime}$ & 0.0018 & 1.17 & 0.0021 \\
Oct. 08 & $i^{\prime}$ & 0.0019 & 1.29 & 0.0025 \\
Oct. 08 & $z^{\prime}$ & 0.0020 & 1.33 & 0.0027 \\
\hline
\end{tabular}

Notes. $\sigma$ represents the residual scatter over the entire observing time interval on each run; $\sigma_{\mathrm{sys}}$ is the rescaled value for the photometric weights, reflecting the presence of red noise.

Furthermore, we assumed that the inclination and the semimajor axis of the planetary orbit should not depend on the observed pass-band. Therefore, we constrained these parameters to a single universal value. Following this procedure for the radius of the planet we found $1.413 \pm 0.020 R_{\text {Jup }}$ and the best-fit value for the orbital inclination and semimajor axis $i=88.57^{\circ} \pm 0.45^{\circ}$ and $a=0.02300 \pm 0.00036 \mathrm{AU}$, respectively. For comparison of the key parameter, $R_{\mathrm{p}}$, Wilson et al. (2008) derived $1.416_{-0.043}^{+0.068} R_{\text {Jup }}$, Gillon et al. (2008) derived $1.304_{-0.042}^{+0.054} R_{\text {Jup }}$, Winn et al. (2009) derived $1.365 \pm 0.021 R_{\text {Jup }}$, Southworth et al. (2009) derived $1.371_{-0.035}^{+0.032} R_{\text {Jup }}$ and Sanchis-Ojeda et al. (2011) derived $1.363 \pm 0.020 R_{\text {Jup }}$. Our value for $R_{\mathrm{p}}$ is slightly higher than the cited radius in the literature and is dominated by the uncertainty of the stellar radius $R_{*}$. The value for the orbital inclination from our analysis agrees well with earlier results. For example, Winn et al. (2009) found $88.56_{+0.98}^{\circ-0.46}$, Sanchis-Ojeda et al. (2011) found $88.80^{\circ-0.43}$, while Gillon et al. (2009) and Soutworth et al. (2009) found $89.35_{-0.49}^{\circ+0.64}$ and $88.80^{\circ}-90.00^{\circ}$, respectively. The results for the fitted parameters of the transit light curve modeling allow us to derive a set of physical properties for the WASP-4 system. Table 3 exhibits a complete list of these quantities.

To complete the light curve analysis we further-more fitted the light curves allowing the linear and the quadratic limbdarkening coefficients $\left(u_{1}\right.$ and $\left.u_{2}\right)$ in each pass-band to be treated as free parameters. We aim to compare the shapes of the transit light curves and the fitted system parameters from this fit and the curves derived using theoretically predicted limb-darkening coefficients. To minimize the $\chi^{2}$-statistic using all 11 parameters, including eight limb-darkening coefficients we again employed the downhill simplex algorithm and the bootstrap method. The new parameter values we find $R_{\mathrm{p}}=1.409 \pm 0.020 R_{\mathrm{Jup}}, i=$ $88.75^{\circ} \pm 0.40^{\circ}, a=0.02298 \pm 0.00033 \mathrm{AU}$ result in a slightly lower value of the $\chi_{\text {red }}^{2}$ function (1.001), because the fit is able to remove some trends in the data. However, we report the parameter values derived using the theoretically predicted limbdarkening coefficient as final results because the new fit is also more sensitive to systematics in the light curve photometry.

A set of multiband transit light curves also allows one to search for a dependence of the planetary radius, $R_{\mathrm{p}}$ as a function of the wavelength. Variations of $R_{\mathrm{p}}$ in some of the pass-bands might be produced by absorption lines such as water vapor,
Table 5. Best-fit radius in each band.

\begin{tabular}{lcc}
\hline \hline Band & $\begin{array}{c}\lambda \\
(\mathrm{nm})\end{array}$ & $\begin{array}{c}\text { Radius } \\
R_{\text {Jup }}\end{array}$ \\
\hline$g^{\prime}$ & 455 & $1.409 \pm 0.021$ \\
$r^{\prime}$ & 627 & $1.415 \pm 0.020$ \\
$i^{\prime}$ & 763 & $1.407 \pm 0.021$ \\
$z^{\prime}$ & 893 & $1.420 \pm 0.021$ \\
\hline
\end{tabular}

Notes. The best-fit radii were derived after fixing the orbital inclination and normalized semimajor axis to their best-fit values from Table 3 .

methane, etc. in the planetary atmosphere. As a check for this assumption we individually fitted the data originating from each pass-band. Instead of fixing $R_{\mathrm{p}} / R_{*}, i$ and $a / R_{*}$ to a a single universal value, we set the planet to star radius ratio as a free parameter and kept the remaining quantities as free parameters. Table 5 displays the best-fit radii as a function of the wavelength with no indications of variations within the measured errorbars.

\subsection{Deriving the transit ephemeris}

One of our primary goals is to measure an accurate value for the transit ephemeris $\left(T_{0}\right.$ and $P$ ). We included all available light curves from the three runs and fitted for the locations of minimum light using the best-fit planetary radius, inclination, and semimajor axis from Sect. 4.1. We minimized the $\chi^{2}$ as defined in Sect. 4 over the four-band data of each run by fitting simultaneously for $T_{\mathrm{C}}$. After the best-fit transit mid-times were derived, we added them to all reported transit mid-times available at the time of writing in the literature ${ }^{2}$ (Table 5). We also fitted for the orbital period $P$ and the reference transit epoch $T_{0}$ by plotting the transit mid-times as a function of the observed epoch $(E)$

$T_{\mathrm{C}}(E)=T_{0}+E \times P$.

In this linear fit the constant coefficient is the best-fit reference transit epoch $\left(T_{0}\right)$ and the slope of the line is the planetary period, $P$. We then used the new values for the period and the reference epoch to repeat our fitting procedure for the system parameters until we arrived at a point of convergence. We used the online converter developed by Eastman et al. (2010) and transformed the transit mid-times from JD based on UTC to BJD based on the barycentric dynamical time (TDB). We derived a period $=1.33823144 \pm 0.00000032$ day and a reference transit time $T_{\mathrm{C}}=2454697.798311 \pm 0.000046 \mathrm{BJD}$. The result for the best-fit period is consistent with that of Sanchis-Ojeda et al. (2011), who found $P=1.33823187 \pm 0.00000025$ day, and reported a smaller error because they measured the period using all available transit times, including the four additional measurements reported by Southworth et al. (2009).

We further more investigated the observed minus calculated $(\mathrm{O}-\mathrm{C})$ residuals of our data and the reported transit mid-times at the time of writing for any departures from the predicted values estimated using our ephemeris. As was shown by Holman \& Murray (2005) and Agol et al. (2005), a deviation in the O-C values can potentially reveal the presence of moons or additional planets in the system. We list and plot the $\mathrm{O}-\mathrm{C}$ values from our analysis in Table 5 and Fig. 3, respectively. An analysis of the

\footnotetext{
2 Southwort et al. (2009) measured mid-times during four transits of WASP-4b. We excluded these times from our analysis, because they were reported as unreliable owing to technical problems associated with the computer clock at the time of observations (Southworth 2011, priv. comm.)
} 
N. Nikolov et al.: WASP-4b transit observations with GROND
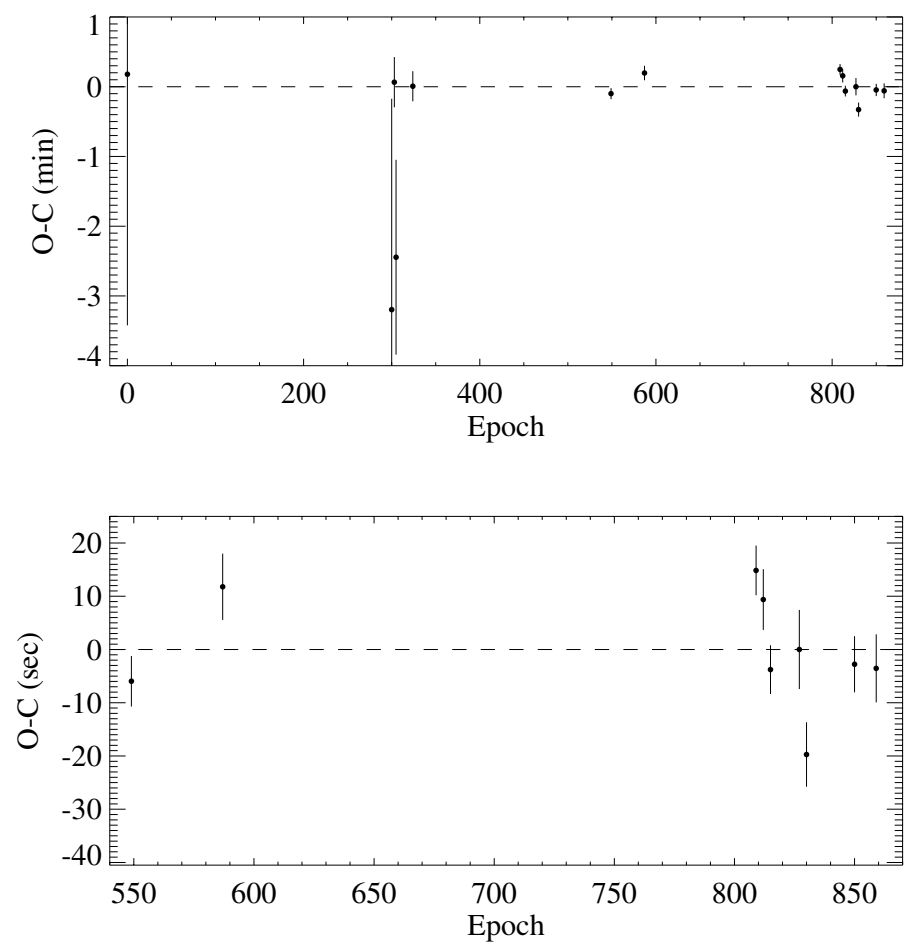

Fig. 3. Transit timing residuals for WASP- $4 b$ along with the one sigma errorbars. Top panel: the observed transit mid-times based on this workand others in the literature were subtracted from the calculated times produced by our ephemeris. Lower panel: a closer view of the available transit mid-times from 2009 and 2010.

O-C values shows no sign of a TTV signal greater than $20 \mathrm{~s}$, except for the two big outliers at epochs 300 and 305. We defived upper constraints on the mass of an aditional perturbing planet in the system as a function of its orbital parameters. We simplified the three-body problem by assumimg that the system is coplanar and initial orbits of both planets are circular. The orbital period of the perturber was varied in a range between 0.1 and 10 orbital periods of WASP-4b. We generated 1000 synthetic O-C diagrams based on calculations computed with the MERCURY package (Chambers 1999). We applied the Bulirsch-Stoer algorithm to integrate the equations of motion. Calculations covered 1150 days, i.e. 860 periods of the transiting planet, that are covered by observations. The results of simulations are presented in Fig. 4. Our analysis allows us to exclude additional Earth-mass planets close to low-order period commensurabilities with WASP-4b.

For a transiting planet with a semi major axis $a_{1}$ and period $P_{1}$ and a perturbing planet with semi major axis $a_{2}$ on an outer orbit (i.e. $a_{2} \geq a_{1}$ ), period $P_{2}$ and mass $M_{2}$ Holman \& Murray (2005) derived the approximate formula

$$
\begin{aligned}
\Delta t & \simeq \frac{45 \pi}{16}\left(\frac{M_{2}}{M_{*}}\right) P_{1} \alpha_{e}^{3}\left(1-\sqrt{2} \alpha_{e}^{3 / 2}\right)^{-2} \\
\alpha_{e} & =\frac{a_{1}}{a_{2}\left(1-e_{2}\right)}
\end{aligned}
$$

for the magnitude of the variation (in seconds) of the time interval $(\Delta t)$ between successive transits. One could imagine an exterior perturbing planet on a circular coplanar orbit twice as far as WASP-4b (period $P \approx 3.75$ day not in mean motion resonance with the transiting planet). If such an imaginary planet had a mass of $0.1 M_{\text {Jup }}$, it would have induced variations of about $5 \mathrm{~s}$ in the predicted transit mid-times of WASP-4b. Furthermore, the perturber could cause radial velocity variations of the parent star
Table 6. Literature transit mid-times of WASP-4 and their residuals in addition to the ephemeris derived in this work.

\begin{tabular}{lccc}
\hline \hline Epoch & $\begin{array}{c}\text { Transit midtime } \\
(\mathrm{BJD})\end{array}$ & $\begin{array}{c}\text { O-C } \\
\text { (day) }\end{array}$ & Reference \\
\hline 0 & $2453963.1094_{-0.0021}^{+0.0025}$ & 0.00011 & 1 \\
300 & $2454364.5765_{-0.0033}^{+0.0021}$ & -0.00223 & 1 \\
303 & $2454368.59341_{-0.00027}^{+0.00025}$ & 0.00003 & 1 \\
305 & $2454371.26813_{-0.00007}^{+0.00097}$ & -0.00171 & 1 \\
324 & $2454396.69623_{-0.00026}^{+0.00015}$ & -0.00001 & 1 \\
549 & $2454697.798228_{-0.000055}^{+0.000055}$ & -0.00008 & 2 \\
587 & $2454748.651228_{-0.0000072}^{+0.000072}$ & 0.00012 & 2 \\
809 & $2455045.738643_{-0.000054}^{+0.000054}$ & 0.00016 & 3 \\
812 & $2455049.753274_{-0.000066}^{+0.00006}$ & 0.00010 & 3 \\
815 & $2455053.767816_{-0.000053}^{+0.000053}$ & -0.00006 & 3 \\
827 & $2455069.826637_{-0.000086}^{+0.000006}$ & -0.00001 & 4 \\
830 & $2455073.841103_{-0.0000070}^{+0.000070}$ & -0.00024 & 4 \\
850 & $2455100.605928_{-0.000061}^{+0.000061}$ & -0.00005 & 3 \\
859 & $2455112.650002_{-0.000074}^{+0.000074}$ & -0.00005 & 4 \\
\hline
\end{tabular}

Notes. (1) Gillon et al. (2009); (2) Winn et al. (2009); (3) Sanchis-Ojeda et al. (2011); (4) this work; the epoch of the first observed transit of WASP-4b was taken to be equal to zero.

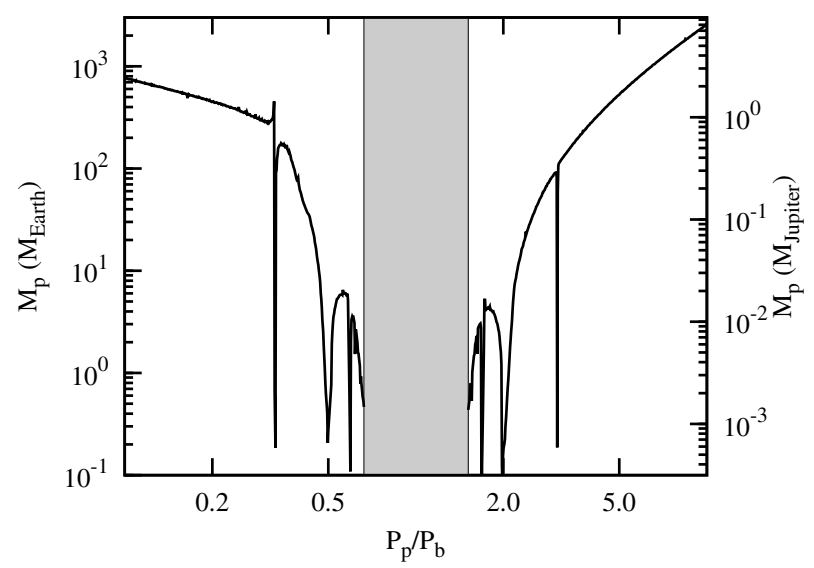

Fig. 4. Upper-mass limit of a hypothetical additional planet that could perturb the orbital motion of WASP-4b as a function of ratio of orbital periods of transiting planet, $P_{\mathrm{b}}$, and the perturber, $P_{\mathrm{p}}$. Orbits located in a gray area were found to be unstable.

$\sim 13.78 \mathrm{~m} / \mathrm{s}$, which is below the radial velocity rms of the WASP4 residual equal to $15.16 \mathrm{~m} / \mathrm{s}$, presented in the analysis of Triaud et al. (2010). Although a few outliers are visible in the $\mathrm{O}-\mathrm{C}$ diagram, we consider the prediction of such an imaginary planet in the WASP-4 system as premature, because the weights of the $\mathrm{O}-\mathrm{C}$ points might still require an additional term to account for systematics of unknown origin.

\section{Concluding remarks}

We have used the GROND multi-channel instrument to obtain four-band simultaneous light curves of the WASP-4 system during three transits (a total of 12 light curves) with the aim to refine the planet and star parameters and to search for transit timing variations. We derived the final values for the planetary 
radius $R_{\mathrm{p}}$ and the orbital inclination $i$ by fixing the stellar radius $R_{*}$ and mass $M_{*}$ to the independently derived values of Winn et al. (2009) and Sanchis-Ojeda et al. (2011). We included the time-correlated "red-noise" in the photometric uncertainties using the "time-averaging" methodology and and by rescaling the weights to produce a value for the $\chi_{\text {red }}^{2}$ equal to unity. We also performed the light curve analysis by minimizing the $\chi^{2}$ function over all pass-bands and runs simultaneously via two approaches. First we modeled the data using theoretically predicted limbdarkening coefficients for the quadratic law. Second, we fitted the light curves for the limb-darkening. Both methods resulted in consistent system parameters within $<1 \%$. The second method produced limb-darkening parameters compatible with the theoretical predictions within the one-sigma errorbars of the fitted parameters.

We added three new transit mid-times for WASP-4b, derived a new ephemeris and investigated the $\mathrm{O}-\mathrm{C}$ diagram for outlier points. We have not found compelling evidence for outliers that could be produced by the presence of a second planet in the system. We did not detect any short-lived photometric anomalies such as occultations of starspots by the planet, which where detected by Sanchis-Ojeda et al. (2011). At the transit rms level of our light curves ( 2.2 mmag) it would be challenging to detect similar anomalies. However, we note that due to the brightness of the parent star, the short planetary orbital period and the significant transit depth, the WASP-4 system is well-suited for follow-up observations.

Acknowledgements. Part of the funding for GROND (both hardware as well as personnel) was generously granted from the Leibniz-Prize to Prof. G. Hasinger (DFG grant HA 1850/28-1). N.N. acknowledges the Klaus Tschira Stiftung (KTS) and the Heidelberg Graduate School of Fundamental Physics (HGSFP) for the financial support of his Ph.D. research. G.M. acknowledges the financial support from the Polish Ministry of Science and Higher Education through the Iuventus Plus grant IP2010 023070. The authors acknowledge Luigi Mancini, John Southworth and the anonymous referee for their useful comments and suggestions.

\section{References}

Agol, E., Steffen, J., Sari, R., \& Clarkson, W. 2005, MNRAS, 359, 567

Beerer, I. M., Knutson, H. A., Burrows, A., et al. 2011, ApJ, 727, 23

Cáceres, C., Ivanov, V. D., Minniti, D., et al. 2011, A\&A, 530, A5

Chambers, J. E. 1999, MNRAS, 304, 793

Charbonneau, D., Brown, T. M., Latham, D. W., \& Mayor, M. 2000, ApJ, 529, L45

Claret, A. 2004, A\&A, 428, 1001

Eastman, J., Siverd, R., \& Gaudi, B. S. 2010, PASP, 122, 935

Gibson, N. P., Pollacco, D., Simpson, E. K., et al. 2008, A\&A, 492, 603

Gillon, M., Pont, F., Moutou, C., et al. 2006, A\&A, 459, 249

Gillon, M., Smalley, B., Hebb, L., et al. 2009, A\&A, 496, 259

Greiner, J., Bornemann, W., Clemens, C., et al. 2008, PASP, 120, 405

Grillmair, C. J., Charbonneau, D., Burrows, A., et al. 2007, ApJ, 658, 115

Henry, G. W., Marcy, G. W., Butler, R. P., \& Vogt, S. S. 2000, ApJ, 529, 41

Holman, M. J., \& Murray, N. W. 2005, Science, 307, 1288

Holman, M. J., Fabrycky, D. C., Ragozzine, D., et al. 2010, Science, 330, 6000

Jha, S., Charbonneau, D., Garnavich, P. M., et al. 2000, ApJ, 540, 45J

Johnson, J. A., Winn, J. N., Bakos, G. Á., et al. 2011, ApJ, 735, 24

Knutson, H. A., Charbonneau, D., Noyes, R. W., Brown, T. M., \& Gilliland, R. L. 2007, ApJ, 655, 564

Lissauer, J. J., Fabrycky, D. C., Ford, E. B., et al. 2011, Nature, 470, 53

Mandel, K., \& Agol, E. 2002, ApJ, 580, L171

Mazeh, T., Naef, D., Torres, G., et al. 2000, ApJ, 532, L55

Miralda-Escudé, J. 2002, ApJ, 564, 1019

Pollacco, D. L., Skillen, I., Collier Cameron, A., et al. 2006, PASP, 118, 1407

Pont, F., Zucker, S., \& Queloz, D. 2006, MNRAS, 373, 231

Press, W. H., Teukolsky, S. A., Vetterling, W. T., \& Flannery, B. P. 1992, Numerical recipes in C, et al. The art of scientific computing, ed. W. H. Press Queloz, D., Eggenberger, A., Mayor, M., et al. 2000, A\&A, 359, 13

Richardson, L. J., Deming, D., Horning, K., Seager, S., \& Harrington, J. 2007, Nature, 445, 892

Sanchis-Ojeda, R., Winn, J. N., Holman, M. J., et al. 2011, ApJ, 733, 127

Southworth, J., Hinse, T. C., Burgdorf, M. J., et al. 2009, MNRAS, 399, 287

Triaud, A. H. M. J., Collier Cameron, A., Queloz, D., et al. 2010, A\&A, 524, A25

Winn, J. N., Holman, M. J., Henry, G. W., et al. 2007, AJ, 133, 1828

Winn, J. N., Holman, M. J., Torres, G., et al. 2008, ApJ, 683, 1076

Winn, J. N., Holman, M. J., Carter, J. A., et al. 2009, AJ, 137, 3826

Winn, J. N., Howard, A. W., Johnson, J. A., et al. 2011, AJ, 141, 63

Wilson, D. M., Gillon, M., Hellier, C., et al. 2008, ApJ, 675, L113 